\title{
T-CONVERGENCE OF FUNCTIONALS ON DIVERGENCE-FREE FIELDS
}

\author{
NAdia Ansini ${ }^{1}$ And Adriana Garroni ${ }^{2}$
}

\begin{abstract}
We study the stability of a sequence of integral functionals on divergence-free matrix valued fields following the direct methods of $\Gamma$-convergence. We prove that the $\Gamma$-limit is an integral functional on divergence-free matrix valued fields. Moreover, we show that the $\Gamma$-limit is also stable under volume constraint and various type of boundary conditions.
\end{abstract}

Mathematics Subject Classification. 35E99, 35J99, 49J45.

Received January 18, 2006.

Published online September 5, 2007.

\section{INTRODUCTION}

In the setting of continuum mechanics and electromagnetism it is interesting to consider variational models involving integral functionals depending on fields which satisfy a differential constraint.

In many applications the constraint of being a solenoidal field is of particular interest. This is the type of functionals we have in mind:

$$
F(U, \Omega)= \begin{cases}\int_{\Omega} f(x, U) \mathrm{d} x & U \in L^{p}\left(\Omega ; \mathbb{M}^{d \times n}\right), \operatorname{Div} U=0 \text { in } \Omega \\ +\infty & \text { otherwise, }\end{cases}
$$

where the function $f$ is a Carathéodory function satisfying the following growth condition

$$
\alpha\|\Sigma\|^{p}-\frac{1}{\alpha} \leq f(x, \Sigma) \leq \beta\left(\|\Sigma\|^{p}+1\right)
$$

for every $x \in \Omega$ and $\Sigma \in \mathbb{M}^{d \times n}$, and $\operatorname{Div} U$ denotes the differential constraint which acts on a $d \times n$ matrix valued function by computing the divergence of each row. Functionals of this type arise naturally from problems in elasticity or, more generally by duality, from problems depending on gradient fields, as for instance in the study of bounds for effective properties of composite materials (see for instance [11-13]).

The problem of characterizing the lower semicontinuity and the relaxation for functionals of type (1.1) has been addressed by [7] and [5] in the context of $\mathcal{A}$-quasiconvexity; i.e., for more general differential constraints $\mathcal{A} U=0$, where $\mathcal{A}$ is a first order linear partial differential operator of constant rank (see Murat [9] and Tartar $[14])$.

\footnotetext{
Keywords and phrases. $\mathcal{A}$-quasiconvexity, divergence-free fields, $\Gamma$-convergence, homogenization.

1 Section de Mathématiques, EPFL, 1015 Lausanne, Switzerland; nadia.ansini@epfl.ch

2 Dip. di Matematica, Univ. di Roma 'La Sapienza', P.le A. Moro 2, 00185 Rome, Italy; garroni@mat.uniroma1.it
}

(c) EDP Sciences, SMAI 2007 
The particular case $\mathcal{A}=\operatorname{div}$ (with $d=1$ ) has been considered in [10]. In [5] it is also proved an homogenization result in terms of $\Gamma$-convergence of functionals

$$
F_{\varepsilon}(U, \Omega)= \begin{cases}\int_{\Omega} f\left(\frac{x}{\varepsilon}, U\right) \mathrm{d} x & U \in L^{p}\left(\Omega ; \mathbb{R}^{N}\right), \mathcal{A} U=0 \text { in } \Omega \\ +\infty & \text { otherwise }\end{cases}
$$

with a general first order differential operator $\mathcal{A}$ of constant rank.

The approach in [5] is very general and is given in terms of Young measures. A key argument relies on a result of decomposition of converging sequences due to [7] in the spirit of the equintegrability result of Acerbi and Fusco [1] and Fonseca, Müller and Pedregal [8].

Our aim is to show that in case of divergence free fields this approach can be simplified and a more explicit construction can be given. Our method is based on a localization and representation technique and can be used more generally for the study of $\Gamma$-convergence of an arbitrary sequence of functionals of type (1.1) and not only in case of homogenization. In order to use a representation argument, as usual, it is essential to prove the additivity and the inner regularity of the limit functional with respect to the domain. To this end the main difficulty is to glue two divergence free fields by keeping the divergence constraint. We do this by modifying the fields in a nonlocal manner by means of an auxiliary system of partial differential equations. This construction implicitly is done in abstract in [5] and [7], however we believe that it can be interesting to give it explicitly. In particular, the explicit construction permits to deal with additional constraints as boundary conditions.

The plan of the paper is the following. In Section 2 we briefly recall the notions of Div-quasiconvexity and $\Gamma$-convergence and we state the main result of the paper.

Section 3 is devoted to the main step in our proof, the so called fundamental estimate, that permits to prove, in Section 4, the compactness and integral representation results and analyze, in Section 5, various boundary value problems.

Finally, we conclude showing that the special case of homogenization can be obtained as a particular case of our general $\Gamma$-convergence result.

\section{Notation, PRELiminaries AND the Main RESUlt}

Let $\Omega$ be a bounded open subset of $\mathbb{R}^{n}$. Let $\Sigma \in \mathbb{M}^{d \times n}$, where $\mathbb{M}^{d \times n}$ stands for the space of $d \times n$ real matrices, we denote $\|\Sigma\|=\sum_{i=1}^{d}\left|(\Sigma)_{i}\right|$, where $(\Sigma)_{i}$ is the $i$ th row of $\Sigma$ and $\left|(\Sigma)_{i}\right|$ its euclidean norm. We use $(z)_{i}$ also to denote the $i$ th component of a vector $z$. The notation $\Sigma \cdot a$ stands for the matrix $\Sigma \in \mathbb{M}^{d \times n}$ that acts on the vector $a \in \mathbb{R}^{n}$ while $\langle\cdot, \cdot\rangle$ denotes the scalar product between two vectors. Finally, we define $\operatorname{Div} \Sigma: \Omega \mapsto \mathbb{R}^{d}$ such that

$$
(\operatorname{Div} \Sigma)_{i}=\operatorname{div}(\Sigma)_{i}
$$

for every $i=1, \ldots, d$.

Our aim is to study the asymptotic behaviour of sequences of integral functionals on divergence free matrix valued fields of the following type

$$
F_{j}(U)= \begin{cases}\int_{\Omega} f_{j}(x, U) \mathrm{d} x & U \in L^{p}\left(\Omega ; \mathbb{M}^{d \times n}\right), \operatorname{Div} U=0 \text { in } \Omega \\ +\infty & \text { otherwise }\end{cases}
$$

where $f_{j}: \Omega \times \mathbb{M}^{d \times n} \mapsto[0, \infty)$ is a sequence of Borel functions satisfying the following conditions:

$$
\exists \alpha, \beta>0 \quad \text { such that } \quad \alpha\|\Sigma\|^{p}-\frac{1}{\alpha} \leq f_{j}(x, \Sigma) \leq \beta\left(\|\Sigma\|^{p}+1\right)
$$


for every $j \in \mathbb{N}, x \in \Omega$ and $\Sigma \in \mathbb{M}^{d \times n}$;

$$
\left|f_{j}\left(x, \Sigma^{1}\right)-f_{j}\left(x, \Sigma^{2}\right)\right| \leq \omega\left(\left\|\Sigma^{1}-\Sigma^{2}\right\|, \Sigma^{1}, \Sigma^{2}\right)
$$

for every $j \in \mathbb{N}, x \in \Omega$ and $\Sigma^{1}, \Sigma^{2} \in \mathbb{M}^{d \times n}$, with

$$
\omega\left(\left\|\Sigma^{1}-\Sigma^{2}\right\|, \Sigma^{1}, \Sigma^{2}\right)=\gamma\left\|\Sigma^{1}-\Sigma^{2}\right\|\left(\left\|\Sigma^{1}\right\|^{p-1}+\left\|\Sigma^{2}\right\|^{p-1}+1\right)
$$

for a given $\gamma>0$.

The notion of convergence, that we will use for $\left\{F_{j}\right\}$, is the, by now classic, notion of $\Gamma$-convergence introduced by De Giorgi. Together with a compactness result for sequences with equibounded energies this is the suitable notion in order to get convergence of minima and minimizing sequences (for a comprehensive study of $\Gamma$ convergence see for instance [6] or [3]). Therefore in view of the a priori bound (2.5) the natural topology use in the study of the $\Gamma$-limit of $\left\{F_{j}\right\}$ is the weak $L^{p}$ topology.

Our main result is that the class of functionals of the type (1.1) is closed under the $\Gamma$-convergence with respect to the weak $L^{p}$ convergence. Namely, we show that given the sequence of functionals defined by (2.4) there exist a subsequence (still labelled with $\left\{F_{j}\right\}$ ) and a functional $F$ of the same type, such that for every $U \in L^{p}\left(\Omega, \mathbb{M}^{d \times n}\right)$, with $\operatorname{Div} U=0$, the following conditions are satisfied:

(i) for every sequence $\left\{U_{j}\right\}$, with $\operatorname{Div} U_{j}=0$, converging to $U$ weakly in $L^{p}$ we have

$$
F(U) \leq \liminf _{j \rightarrow+\infty} F_{j}\left(U_{j}\right)
$$

(ii) there exists a sequence $\left\{U_{j}\right\}$, with $\operatorname{Div} U_{j}=0$, converging to $U$ weakly in $L^{p}$ such that

$$
F(U) \geq \limsup _{j \rightarrow+\infty} F_{j}\left(U_{j}\right)
$$

It is well known that a $\Gamma$-limit with respect to a given topology is always lower semicontinuous in that topology. The lower semicontinuity and the relaxation have been characterized in [7] and [5] in the general case of integral functionals satisfying a differential constraint given by a first order linear partial differential operator $\mathcal{A}$ of constant rank (see Murat [9] and Tartar [14]).

In the particular case of divergence constraint the necessary and sufficient condition for the lower semicontinuity of functionals of type (1.1) is given by the Div-quasiconvexity of $f(x, \cdot)$ for a.e. $x \in \Omega$. We say that a function $g: \mathbb{M}^{d \times n} \mapsto \mathbb{R}$, satisfying $\alpha\|\Sigma\|^{p}-1 / \alpha \leq g(\Sigma) \leq \beta\left(\|\Sigma\|^{p}+1\right)$ for some $\alpha, \beta>0$, is Div-quasiconvex if for every $\Sigma \in \mathbb{M}^{d \times n}$

$$
g(\Sigma)=\inf \left\{\int_{Q} g(\Sigma+V(x)) \mathrm{d} x: V \in L_{\#, \text { Div }}^{p}\left(Q ; \mathbb{M}^{d \times n}\right), \int_{Q} V \mathrm{~d} x=0\right\}
$$

where

$$
L_{\#, \text { Div }}^{p}\left(Q ; \mathbb{M}^{d \times n}\right)=\left\{U \in L_{\text {loc }}^{p}\left(\mathbb{R}^{n}, \mathbb{M}^{d \times n}\right): Q \text {-periodic, Div } U=0 \text { in } \mathbb{R}^{n}\right\},
$$

and $Q$ denotes the unit cube in $\mathbb{R}^{n}$.

A necessary condition for the Div-quasiconvexity is given by the rank- $(n-1)$ convexity (see [7], Prop. 3.4). Here we say that a function $g: \mathbb{M}^{d \times n} \mapsto \mathbb{R}(d \geq n)$ is rank- $(n-1)$ convex if for all $\Sigma^{1}, \Sigma^{2} \in \mathbb{M}^{d \times n}$ such that $\operatorname{rank}\left(\Sigma^{1}-\Sigma^{2}\right) \leq(n-1)$

for all $t \in(0,1)$.

$$
g\left(t \Sigma^{1}+(1-t) \Sigma^{2}\right) \leq t g\left(\Sigma^{1}\right)+(1-t) g\left(\Sigma^{2}\right)
$$

Remark 2.1. If $d<n$ the notion of $\operatorname{rank}-(n-1)$ convexity coincides with convexity.

Moreover, if $d=1$ the div-quasiconvexity reduces to convexity. In this case, the relaxation of a functional of the form (1.1) is represented by the convex envelope of $f$ with respect to the second variable; i.e., the div-quasiconvex envelope $Q_{\operatorname{div}} f(x, \cdot)$ coincides with $f^{* *}(x, \cdot)$ (see [5] and [10] and also Rem. 3.5 (iv) in [7]). 
Our main result can be stated as follows.

Theorem 2.2. For any sequence of functionals $\left\{F_{j}\right\}$ defined by (2.4) and satisfying (2.5) and (2.6) there exist a subsequence $\left\{F_{j_{k}}\right\}$ and a Borel function $\varphi: \Omega \times \mathbb{M}^{d \times n} \mapsto[0,+\infty)$, Div-quasiconvex in the second variable, satisfying the condition

$$
\alpha\|\Sigma\|^{p}-\frac{1}{\alpha} \leq \varphi(x, \Sigma) \leq \beta\left(1+\|\Sigma\|^{p}\right)
$$

such that $\left\{F_{j_{k}}\right\} \Gamma$-converges with respect to the weak $L^{p}$ topology to

$$
F(U)= \begin{cases}\int_{\Omega} \varphi(x, U) \mathrm{d} x & U \in L^{p}\left(\Omega ; \mathbb{M}^{d \times n}\right), \operatorname{Div} U=0 \text { in } \Omega \\ +\infty & \text { otherwise. }\end{cases}
$$

Remark 2.3. Note that we assume the Lipschitz condition (2.6) in order to simplify the argument, but this is not at all restrictive. Indeed in the study of the $\Gamma$-limit of $\left\{F_{j}\right\}$ it is always possible to assume that $F_{j}$ are lower semicontinuous with respect to the weak $L^{p}$ topology (if not we can replace the sequence $\left\{F_{j}\right\}$ with the sequence of the corresponding relaxed functionals). Thus, in view of the characterization of the lower semicontinuous functionals, we may always assume that $\left\{f_{j}\right\}$ are Div-quasiconvex, hence rank- $(n-1)$ convex. This implies that the growth condition $f_{j}(x, \Sigma) \leq \beta\left(1+\|\Sigma\|^{p}\right)$ is sufficient to conclude the Lipschitz condition

$$
\left|f_{j}\left(x, \Sigma^{1}\right)-f_{j}\left(x, \Sigma^{2}\right)\right| \leq \gamma\left\|\Sigma^{1}-\Sigma^{2}\right\|\left(\left\|\Sigma^{1}\right\|^{p-1}+\left\|\Sigma^{2}\right\|^{p-1}+1\right)
$$

for every $\Sigma^{1}, \Sigma^{2} \in \mathbb{M}^{d \times n}$ and $x \in \Omega$ with $\gamma$ depending on $\beta$ and $p$ (see e.g. [4], Rem. 4.13 (iii)).

For the proof of Theorem 2.2 we will use the classical strategy of localization and integral representation. To this end it is convenient to introduce an explicit dependence of our functionals on the domain. We denote by $\mathcal{A}(\Omega)$ the family of all open subsets of $\Omega$ and for every $j \in \mathbb{N}$ we consider the functionals $F_{j}: L^{p}\left(\Omega ; \mathbb{M}^{d \times n}\right) \times$ $\mathcal{A}(\Omega) \rightarrow \overline{\mathbb{R}}$ given by

$$
F_{j}(U, A)= \begin{cases}\int_{A} f_{j}(x, U) \mathrm{d} x & U \in L^{p}\left(\Omega ; \mathbb{M}^{d \times n}\right), \operatorname{Div} U=0 \text { in } \Omega \\ +\infty & \text { otherwise }\end{cases}
$$

Let us finally recall that this strategy also requires the characterization of the $\Gamma$-convergence in terms of the lower and upper $\Gamma$-limit, i.e.,

$$
F^{\prime}(U, A)=\Gamma-\liminf _{j \rightarrow+\infty} F_{j}(U, A)=\inf \left\{\liminf _{j \rightarrow+\infty} F_{j}\left(U_{j}, A\right): U_{j} \rightarrow U\right\}
$$

and

$$
F^{\prime \prime}(U, A)=\Gamma-\limsup _{j \rightarrow+\infty} F_{j}(U, A)=\inf \left\{\limsup _{j \rightarrow+\infty} F_{j}\left(U_{j}, A\right): U_{j} \rightarrow U\right\} .
$$

Thus the equality $F^{\prime}(U, A)=F^{\prime \prime}(U, A)$ is equivalent to the existence of the $\Gamma$ - $\operatorname{limit} F(U, A)=\Gamma$ - $\lim _{j \rightarrow+\infty} F_{j}(U, A)$.

In the sequel the letter $c$ will denote a positive constant, independent of the parameters under consideration, whose value may vary from line to line.

\section{The Fundamental estimate}

In this section we prove the main tool for our $\Gamma$-convergence result: the fundamental estimate.

Proposition 3.1 (fundamental estimate). Let $p>1$ and let $\left\{F_{j}\right\}$ be a sequence of functionals defined by (2.10) and satisfying conditions (2.5) and (2.6). For every $\sigma>0$, for all $A, A^{\prime}, B \in \mathcal{A}(\Omega)$ with $A^{\prime} \subset \subset A$ and 
$\operatorname{dist}\left(A^{\prime}, B \backslash A\right)>0$, for every $U_{j} \rightarrow U$ and $V_{j} \rightarrow U$ in $L^{p}\left(\Omega ; \mathbb{M}^{d \times n}\right)$ with $\operatorname{Div} U_{j}=\operatorname{Div} V_{j}=\operatorname{Div} U=0$, there exists a sequence $\left\{W_{j}\right\} \subseteq L^{p}\left(\Omega ; \mathbb{M}^{d \times n}\right)$, with $\operatorname{Div} W_{j}=0$, weakly converging to $U$ in $L^{p}\left(\Omega ; \mathbb{M}^{d \times n}\right)$ such that

$$
F_{j}\left(W_{j}, A^{\prime} \cup B\right) \leq F_{j}\left(U_{j}, A\right)+F_{j}\left(V_{j}, B\right)+\sigma+o(1)
$$

as $j \rightarrow+\infty$.

Proof. Let $\delta=\operatorname{dist}\left(A^{\prime}, \partial A\right)$, we fix $N \in \mathbb{N}$. For any $k \in\{1, \ldots, N\}$ we define the set

$$
A_{k}=\left\{x \in A: \quad N \operatorname{dist}\left(x, A^{\prime}\right)<k \delta\right\}
$$

and $A_{0}=A^{\prime}$. Let $\varphi^{k}$ be a cut-off function between $A_{k-1}$ and $A_{k} ;$ i.e., $\varphi^{k} \in C_{0}^{\infty}\left(A_{k}\right)$ and $\varphi^{k}=1$ on $A_{k-1}$. In general $\operatorname{Div}\left(\varphi^{k} U_{j}+\left(1-\varphi^{k}\right) V_{j}\right) \neq 0$, but we can modify $\varphi^{k} U_{j}+\left(1-\varphi^{k}\right) V_{j}$ adding a non local perturbation such that the new sequence is divergence free. More precisely, we define

$$
\left(W_{j}^{k}\right)_{i}=\varphi^{k}\left(U_{j}\right)_{i}+\left(1-\varphi^{k}\right)\left(V_{j}\right)_{i}+\left|D\left(g_{j}^{k}\right)_{i}\right|^{p^{\prime}-2} D\left(g_{j}^{k}\right)_{i}
$$

for $i=1, \ldots, d$, where $g_{j}^{k}: \Omega \rightarrow \mathbb{R}^{d}$ is the solution of the Neumann system

$$
\left\{\begin{array}{l}
-\operatorname{div}\left(\left|D\left(g_{j}^{k}\right)_{i}\right|^{p^{\prime}-2} D\left(g_{j}^{k}\right)_{i}\right)=\left\langle\left(U_{j}-V_{j}\right)_{i}, D \varphi^{k}\right\rangle \quad \text { on } \Omega \\
\frac{\partial\left(g_{j}^{k}\right)_{i}}{\partial \nu}=0 \quad \text { on } \partial \Omega \quad i=1, \ldots, d \\
\int_{\Omega} g_{j}^{k} \mathrm{~d} x=0,
\end{array}\right.
$$

where $\nu$ is the outer normal on $\partial \Omega$ and $1 / p+1 / p^{\prime}=1$. If $p^{\prime}<2$ we use the convention

$$
\left|D\left(g_{j}^{k}\right)_{i}\right|^{p^{\prime}-2} D\left(g_{j}^{k}\right)_{i}= \begin{cases}\left|D\left(g_{j}^{k}\right)_{i}\right|^{p^{\prime}-2} D\left(g_{j}^{k}\right)_{i} & \text { if }\left|D\left(g_{j}^{k}\right)_{i}\right|>0 \\ 0 & \text { if }\left|D\left(g_{j}^{k}\right)_{i}\right|=0 .\end{cases}
$$

Note that $\left(U_{j}-V_{j}\right) \cdot D \varphi^{k}=\operatorname{Div}\left(\varphi^{k} U_{j}+\left(1-\varphi^{k}\right) V_{j}\right)$ and the solution of (3.2) exists in view of the fact that

$$
\int_{\Omega}\left\langle\left(U_{j}-V_{j}\right)_{i}, D \varphi^{k}\right\rangle \mathrm{d} x=\int_{\partial \Omega}\left\langle\left(V_{j}\right)_{i}, \nu\right\rangle \mathrm{d} \mathcal{H}^{n-1}=0
$$

for every $i=1, \ldots, d$, since $\operatorname{Div} V_{j}=0$. Hence, by (3.2) we have that $\operatorname{Div} W_{j}^{k}=0$.

By (2.5) and (2.6), we get that

$$
\begin{aligned}
& \int_{A^{\prime} \cup B} f_{j}\left(x, W_{j}^{k}\right) \mathrm{d} x \\
\leq & \int_{A^{\prime} \cup B} f_{j}\left(x, \varphi^{k} U_{j}+\left(1-\varphi^{k}\right) V_{j}\right) \mathrm{d} x+\int_{A^{\prime} \cup B} \omega_{j}^{k}(x) \mathrm{d} x \\
= & \int_{A^{\prime} \cup\left(B \cap A_{k-1}\right)} f_{j}\left(x, U_{j}\right) \mathrm{d} x+\int_{B \backslash A_{k}} f_{j}\left(x, V_{j}\right) \mathrm{d} x+\int_{A^{\prime} \cup B} \omega_{j}^{k}(x) \mathrm{d} x+\int_{C_{k} \cap B} f_{j}\left(x, \varphi^{k} U_{j}+\left(1-\varphi^{k}\right) V_{j}\right) \mathrm{d} x \\
\leq & F_{j}\left(U_{j}, A\right)+F_{j}\left(V_{j}, B\right)+\int_{A^{\prime} \cup B} \omega_{j}^{k}(x) \mathrm{d} x+\int_{C_{k} \cap B} \beta+\beta 2^{p-1}\left(\left\|U_{j}\right\|^{p}+\left\|V_{j}\right\|^{p}\right) \mathrm{d} x
\end{aligned}
$$


where $C_{k}=A_{k} \backslash A_{k-1}$ and

$$
\omega_{j}^{k}(x)=\omega\left(\sum_{i=1}^{d}\left|D\left(g_{j}^{k}\right)_{i}\right|^{p^{\prime}-1}, W_{j}^{k},\left(\varphi^{k} U_{j}+\left(1-\varphi^{k}\right) V_{j}\right)\right) .
$$

We now choose $k$ such that the last term of the inequality (3.3) is small. Indeed, since $\left\{U_{j}\right\}$ and $\left\{V_{j}\right\}$ are bounded in $L^{p}\left(\Omega ; \mathbb{M}^{d \times n}\right)$, we note that

$$
\sum_{k=1}^{N} \int_{C_{k} \cap B} \beta+\beta 2^{p-1}\left(\left\|U_{j}\right\|^{p}+\left\|V_{j}\right\|^{p}\right) \mathrm{d} x \leq \int_{(A \cap B) \backslash A^{\prime}} \beta+\beta 2^{p-1}\left(\left\|U_{j}\right\|^{p}+\left\|V_{j}\right\|^{p}\right) \mathrm{d} x \leq \tilde{c}
$$

and therefore there exists $k_{j} \in\{1, \ldots, N\}$ such that

$$
\int_{C_{k_{j}} \cap B} \beta+\beta 2^{p-1}\left(\left\|U_{j}\right\|^{p}+\left\|V_{j}\right\|^{p}\right) \mathrm{d} x \leq \frac{1}{N} \int_{(A \cap B) \backslash A^{\prime}} \beta+\beta 2^{p-1}\left(\left\|U_{j}\right\|^{p}+\left\|V_{j}\right\|^{p}\right) \mathrm{d} x \leq \frac{\tilde{c}}{N} .
$$

With this choice of $k_{j}$, we define

$$
W_{j}:=W_{j}^{k_{j}} \quad g_{j}:=g_{j}^{k_{j}} \quad \varphi_{j}:=\varphi^{k_{j}} \quad \omega_{j}=\omega_{j}^{k_{j}} .
$$

Summarizing, we have

$$
\left(W_{j}\right)_{i}=\varphi_{j}\left(U_{j}\right)_{i}+\left(1-\varphi_{j}\right)\left(V_{j}\right)_{i}+\left|D\left(g_{j}\right)_{i}\right|^{p^{\prime}-2} D\left(g_{j}\right)_{i}
$$

for $i=1, \ldots, d$, where $\varphi_{j} \in C_{0}^{\infty}(A)$ is a cut-off function between $A^{\prime}$ and $A$ and $g_{j}$ is the solution of the Neumann system

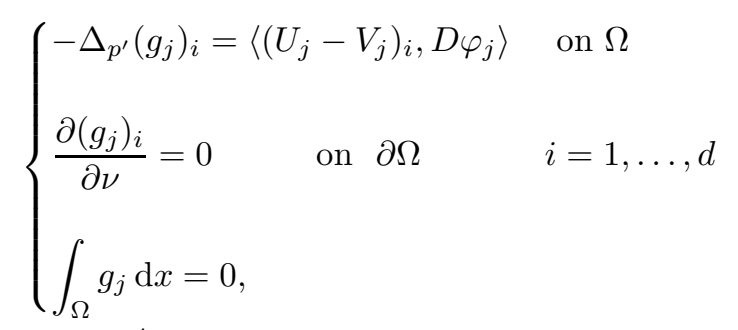

where $1 / p+1 / p^{\prime}=1, \Delta_{p^{\prime}} u=\operatorname{div}\left(|D u|^{p^{\prime}-2} D u\right)$ and $\nu$ is the outer normal on $\partial \Omega$.

From (3.3), in view of (3.4), for every $\sigma>0$, if we fix $N \in \mathbb{N}$ such that $\tilde{c} / N<\sigma$, we get

$$
F_{j}\left(W_{j}, A^{\prime} \cup B\right) \leq F_{j}\left(U_{j}, A\right)+F_{j}\left(V_{j}, B\right)+\sigma+\int_{A^{\prime} \cup B} \omega_{j}(x) \mathrm{d} x .
$$

It remains to prove that

$$
\lim _{j \rightarrow \infty}\left(\int_{A^{\prime} \cup B} \omega_{j}(x) \mathrm{d} x\right)=0 .
$$

By (3.5) and by Hölder's and Poincaré's inequalities, since $\left\{U_{j}\right\}$ and $\left\{V_{j}\right\}$ are bounded in $L^{p}\left(\Omega ; \mathbb{M}^{d \times n}\right)$, we have that

$$
\begin{aligned}
\int_{\Omega}\left|D\left(g_{j}\right)_{i}\right|^{p^{\prime}} \mathrm{d} x & =\int_{\Omega}\left\langle\left(U_{j}-V_{j}\right)_{i}, D \varphi_{j}\right\rangle\left(g_{j}\right)_{i} \mathrm{~d} x \\
& \leq\left(\int_{\Omega}\left|\left\langle\left(U_{j}-V_{j}\right)_{i}, D \varphi_{j}\right\rangle\right|^{p} \mathrm{~d} x\right)^{1 / p}\left(\int_{\Omega}\left|\left(g_{j}\right)_{i}\right|^{p^{\prime}} \mathrm{d} x\right)^{1 / p^{\prime}} \\
& \leq c\left(\int_{\Omega}\left|D\left(g_{j}\right)_{i}\right|^{p^{\prime}} \mathrm{d} x\right)^{1 / p^{\prime}}
\end{aligned}
$$


for every $i=1, \ldots, d$. It follows that $\left\{g_{j}\right\}$ is bounded in the Sobolev space $W^{1, p^{\prime}}\left(\Omega ; \mathbb{M}^{d \times n}\right)$ and, up to a subsequence, it converges strongly in $L^{p^{\prime}}\left(\Omega ; \mathbb{M}^{d \times n}\right)$. Moreover, since $\varphi_{j}$ varies in the finite set $\left\{\varphi_{1}, \ldots, \varphi_{N}\right\}$, all converging subsequences of $\left\{\varphi_{j}\right\}$ are stationary and thus, since $\left(U_{j}-V_{j}\right) \rightarrow 0$ in $L^{p}\left(\Omega ; \mathbb{M}^{d \times n}\right)$, we deduce that

$$
\lim _{j \rightarrow \infty} \int_{\Omega}\left\|D g_{j}\right\|^{p^{\prime}} \mathrm{d} x=0
$$

and hence the strong convergence of $\left\{g_{j}\right\}$ to zero in $W^{1, p^{\prime}}\left(\Omega ; \mathbb{M}^{d \times n}\right)$. Since

$$
\omega_{j}(x) \leq c\left\|D g_{j}\right\|^{p^{\prime}-1}\left(1+\left\|U_{j}\right\|^{p-1}+\left\|V_{j}\right\|^{p-1}+\left\|D g_{j}\right\|\right),
$$

by Hölder's inequality we have that

$$
\int_{\Omega} \omega_{j}(x) \mathrm{d} x \leq c\left(\int_{\Omega}\left\|D g_{j}\right\|^{p^{\prime}} \mathrm{d} x+\left(\int_{\Omega}\left\|D g_{j}\right\|^{p^{\prime}} \mathrm{d} x\right)^{1 / p}\right)
$$

which, in view of (3.7), gives (3.6).

Finally, by the strong convergence of $\left\{g_{j}\right\}$ and the fact that $\left\{\varphi_{j}\right\}$ varies in a finite set of functions, we also deduce that $W_{j} \rightarrow U$ in $L^{p}\left(\Omega ; \mathbb{M}^{d \times n}\right)$.

Remark 3.2. Using the fundamental estimate, by classical arguments, it is possible to prove that the $\Gamma$-lim inf, $F^{\prime}(U, \cdot)$, and the $\Gamma$-limsup, $F^{\prime \prime}(U, \cdot)$, are inner regular increasing set functions and, in particular, $F^{\prime \prime}(U, \cdot)$ is also subadditive (see e.g. [4], Props. 11.5 and 11.6).

In view of the future application of the fundamental estimate to the study of the stability of the $\Gamma$-convergence under average conditions (see Prop. 5.1 and Rem. 5.2) it is convenient to note the following fact.

Remark 3.3. We can restate the fundamental estimate adding a condition on the average. More precisely, for every $\sigma>0$, for all $A, A^{\prime}, B \in \mathcal{A}(\Omega)$ with $A^{\prime} \subset \subset A$ and $\operatorname{dist}\left(A^{\prime}, B \backslash A\right)>0$, taking $\left\{U_{j}\right\},\left\{V_{j}\right\}$ and $U$ as in Proposition 3.1, we can construct a sequence $\left\{W_{j}\right\}$ such that

$$
\left\{\begin{array}{l}
W_{j} \rightarrow U \text { in } L^{p}\left(\Omega ; \mathbb{M}^{d \times n}\right) \\
\operatorname{Div} W_{j}=0 \\
f_{\Omega} W_{j} \mathrm{~d} x=f_{\Omega} U \mathrm{~d} x
\end{array}\right.
$$

and

$$
F_{j}\left(W_{j}, A^{\prime} \cup B\right) \leq F_{j}\left(U_{j}, A\right)+F_{j}\left(V_{j}, B\right)+\sigma+o(1)
$$

as $j \rightarrow+\infty$. The sequence $\left\{W_{j}\right\}$ is explicitly given by

$$
\left(W_{j}\right)_{i}:=\varphi_{j}\left(U_{j}\right)_{i}+\left(1-\varphi_{j}\right)\left(V_{j}\right)_{i}+\left|D\left(g_{j}\right)_{i}\right|^{p^{\prime}-2} D\left(g_{j}\right)_{i}-\left(m_{j}\right)_{i}
$$

for $i=1, \ldots, d$, where $\varphi_{j} \in C_{0}^{\infty}(A)$ is a cut-off function between $A^{\prime}$ and $A, g_{j}$ is the solution of the Neumann system (3.5) and $m_{j} \in \mathbb{M}^{d \times n}$, given by

$$
\left(m_{j}\right)_{i}:=f_{\Omega}\left(\varphi_{j}\left(U_{j}\right)_{i}+\left(1-\varphi_{j}\right)\left(V_{j}\right)_{i}+\left|D\left(g_{j}\right)_{i}\right|^{p^{\prime}-2} D\left(g_{j}\right)_{i}-(U)_{i}\right) \mathrm{d} x
$$

for $i=1, \ldots, d$, converges to zero as $j \rightarrow+\infty$. 


\section{Compactness and integral Representation}

In this section we prove that any sequence $\left\{F_{j}\right\}$ of functionals of the form $(2.10)$ is compact with respect to the $\Gamma$-convergence (Prop. 4.1) and that the $\Gamma$-limit can be represented as an integral functional of the same form (Th. 4.2).

We recall that the compactness of the $\Gamma$-convergence is well known in case of separable metric spaces (see e.g. [4], Th. 7.9). Since we are studying the $\Gamma$-convergence in the $L^{p}$-space with respect to the weak topology, we cannot make use directly of this result.

The proof of the existence of a subsequence $\left\{F_{j_{k}}(\cdot, R)\right\}$ which $\Gamma$-converges to the functional $F(\cdot, R)$ for every $R \in \mathcal{R}(\Omega)$ (union of open cubes contained in $\Omega$ and with vertices in $\mathbb{Q}^{n}$ ) is due to Braides, Fonseca and Leoni (see [5], Lem. 5.3). Fixed $R \in \mathcal{R}(\Omega)$, they essentially use the fact that since $p>1$, the dual of $L^{p}\left(R ; \mathbb{M}^{d \times n}\right)$ is separable and hence the space

$$
l B=\left\{V \in L^{p}\left(R ; \mathbb{M}^{d \times n}\right):\|V\|_{L^{p}} \leq l\right\},
$$

with $l \in \mathbb{N}$, endowed with the weak topology is metrizable. They consider the metric $d_{l}$ which generates the $L^{p}$ weak topology in $l B$ and apply the compactness result for separable metric spaces to the sequence of functionals $\left\{F_{j}(\cdot, R)\right\}$ restricted to the space $\left(l B \cap\{\operatorname{Div}=0\}, d_{l}\right)$. By a recursive procedure on $l$ and a diagonalization process they prove that there exists a subsequence $j_{k}$ such that $\left\{F_{j_{k}}(\cdot, R)\right\} \Gamma$-converges for every $R \in \mathcal{R}(\Omega)$.

In the following proposition we show that, using the fundamental estimate (Prop. 3.1), we can easily extend this compactness result from $\mathcal{R}(\Omega)$ to every open set $A \in \mathcal{A}(\Omega)$.

Proposition 4.1 (compactness). Let $\left\{F_{j}\right\}$ be a sequence of functionals defined by (2.10). Then there exists a subsequence $\left\{F_{j_{k}}\right\}$ such that the $\Gamma$-limit

$$
F(U, A)=\Gamma-\lim _{k \rightarrow+\infty} F_{j_{k}}(U, A)
$$

exists for all $U \in L^{p}\left(\Omega ; \mathbb{M}^{d \times n}\right)$ with $\operatorname{Div} U=0$ and $A \in \mathcal{A}(\Omega)$. Moreover, $F(U, \cdot)$ is the restriction of a Borel measure to $\mathcal{A}(\Omega)$.

Proof. Consider the family $\mathcal{R}(\Omega)$ of all finite unions of open cubes contained in $\Omega$ and with vertices in $\mathbb{Q}^{n}$. By Lemma 5.3 in $[5]$, there exists a subsequence $\left\{j_{k}\right\}$ such that the $\Gamma$-limit

$$
F(U, R)=\Gamma-\lim _{k \rightarrow+\infty} F_{j_{k}}(U, R)
$$

exists for all $R \in \mathcal{R}(\Omega)$ and $U \in L^{p}\left(\Omega ; \mathbb{M}^{d \times n}\right)$ with $\operatorname{Div} U=0$.

Now the extension of the $\Gamma$-convergence of $F_{j_{k}}(U, \cdot)$ to every open set $A \in \mathcal{A}(\Omega)$ is a consequence of the inner regularity of $F^{\prime}(U, \cdot)$ and $F^{\prime \prime}(U, \cdot)$ (see Rem. 3.2) taking into account that if $B \subset \subset A \subseteq \Omega$ then there exists $R \in \mathcal{R}(\Omega)$ such that $B \subset \subset R \subset \subset A$.

Finally, to conclude that $F(U, \cdot)$ is the restriction of a Borel measure to $\mathcal{A}(\Omega)$ it is enough to recall that $F(U, \cdot)$ is an increasing inner regular set function, subadditive and superadditive (see Rem. 3.2) and then to apply the Measure property criterion due to De Giorgi and Letta (see Th. 10.2 in [4]).

Theorem 4.2 (integral representation). Let $\left\{f_{j}\right\}$ be a sequence of Borel functions with $f_{j}: \Omega \times \mathbb{M}^{d \times n} \mapsto[0,+\infty)$ satisfying (2.6) and the growth condition (2.5). Then, there exist a subsequence $j_{k} \rightarrow+\infty$ and a Borel function $\varphi: \Omega \times \mathbb{M}^{d \times n} \mapsto[0,+\infty)$, Div-quasiconvex in the second variable, satisfying the condition

$$
\alpha\|\Sigma\|^{p}-\frac{1}{\alpha} \leq \varphi(x, \Sigma) \leq \beta\left(1+\|\Sigma\|^{p}\right)
$$


such that $\left\{F_{j_{k}}\right\}$, defined by $(2.10)$, $\Gamma$-converges to

$$
F(U, A)= \begin{cases}\int_{A} \varphi(x, U) \mathrm{d} x & U \in L^{p}\left(\Omega ; \mathbb{M}^{d \times n}\right), \operatorname{Div} U=0 \text { in } \Omega, \\ +\infty & \text { otherwise, }\end{cases}
$$

for all $A \in \mathcal{A}(\Omega)$.

Proof. By Proposition 4.1 there exists a subsequence $\left\{F_{j_{k}}\right\}$ which $\Gamma$-converges to $F$. Moreover, by $(2.5), F(U, \cdot)$ can be extended to a Borel measure on $\Omega$ absolutely continuous with respect to the Lebesgue measure. Hence, it remains to deduce the integral representation (4.2). This will be done in several steps. The first three steps use standard arguments that we repeat and adapt to our context for the reader convenience.

Step 1. Definition of $\varphi$.

Let $\Sigma \in \mathbb{M}^{d \times n}$, we denote by $g_{\Sigma} \in L^{1}(\Omega)$ the density of $F(\Sigma, \cdot)$ with respect to the Lesbegue measure; i.e.,

$$
F(\Sigma, A)=\int_{A} g_{\Sigma}(x) \mathrm{d} x
$$

for all $A \in \mathcal{A}(\Omega)$. If we define

$$
\varphi(x, \Sigma)=g_{\Sigma}(x)
$$

for all $x \in \Omega$ and $\Sigma \in \mathbb{M}^{d \times n}$, by (2.5), we get that

$$
\alpha\|\Sigma\|^{p}-\frac{1}{\alpha} \leq \varphi(x, \Sigma) \leq \beta\left(1+\|\Sigma\|^{p}\right)
$$

for a.e. $x \in \Omega$.

Step 2. Integral representation on piecewise constant functions.

Let $A \in \mathcal{A}(\Omega)$ and let $U \in L^{p}\left(\Omega ; \mathbb{M}^{d \times n}\right)$ be a piecewise constant function in $A$, with $\operatorname{Div} U=0$; i.e.,

$$
\left.U\right|_{A}=\sum_{j=1}^{N} \chi_{A_{j}} \Sigma^{j}
$$

where the sets $A_{j}$ are disjoint open sets with $\left|A \backslash \bigcup_{j=1}^{N} A_{j}\right|=0, \Sigma^{j} \in \mathbb{M}^{d \times n}$ for $j=1, \ldots, N$. By Step 1

$$
\begin{aligned}
F(U, A) & =\sum_{j=1}^{N} F\left(U, A_{j}\right)=\sum_{j=1}^{N} F\left(\Sigma^{j}, A_{j}\right)=\sum_{j=1}^{N} \int_{A_{j}} \varphi\left(x, \Sigma^{j}\right) \mathrm{d} x=\sum_{j=1}^{N} \int_{A_{j}} \varphi(x, U) \mathrm{d} x \\
& =\int_{A} \varphi(x, U) \mathrm{d} x .
\end{aligned}
$$

Step 3. Rank- $(n-1)$ convexity of $\varphi$.

By Step 1 we have that

$$
\varphi(x, \Sigma)=\limsup _{\rho \rightarrow 0^{+}} \frac{F\left(\Sigma, B_{\rho}(x)\right)}{\left|B_{\rho}(x)\right|}
$$


for all $x \in \Omega$ and $\Sigma \in \mathbb{M}^{d \times n}$. In view of (2.7) we prove the rank- $(n-1)$ convexity in the second variable of $\varphi$ if, for $B_{\rho}(x) \subset \Omega$, we show that

$$
F\left(t \Sigma^{1}+(1-t) \Sigma^{2}, B_{\rho}(x)\right) \leq t F\left(\Sigma^{1}, B_{\rho}(x)\right)+(1-t) F\left(\Sigma^{2}, B_{\rho}(x)\right)
$$

for all $t \in(0,1)$ and for every $\Sigma^{1} \neq \Sigma^{2} \in \mathbb{M}^{d \times n}$ with $\operatorname{rank}\left(\Sigma^{1}-\Sigma^{2}\right) \leq(n-1)$.

As a consequence of the rank property of $\Sigma^{1}$ and $\Sigma^{2}$ we have that there exists a unit vector $\nu \in \mathbb{R}^{n}$ such that $\left(\Sigma^{1}-\Sigma^{2}\right) \cdot \nu=0$. Hence, if we define $V: \mathbb{R}^{n} \mapsto\left\{\Sigma^{1}, \Sigma^{2}\right\}$ as

$$
V(y)= \begin{cases}\Sigma^{1} & y \in A_{1} \\ \Sigma^{2} & y \in A_{2}\end{cases}
$$

with

$$
\begin{gathered}
A_{1}=\left\{y \in \mathbb{R}^{n}: j<\langle y, \nu\rangle<j+t, j \in \mathbb{Z}^{n}\right\}, \\
A_{2}=\left\{y \in \mathbb{R}^{n}: j+t<\langle y, \nu\rangle<j+1, j \in \mathbb{Z}^{n}\right\}
\end{gathered}
$$

and $t \in(0,1)$, we clearly have $\operatorname{Div} V=0$. Roughly speaking, $A_{1} \cup A_{2}$ represents a lamination of $\mathbb{R}^{n}$ with proportion $t$ and $1-t$, respectively, in the direction orthogonal to $\nu$. We now define $U_{h}(y)=V(h y)$ for $y \in \mathbb{R}^{n}$. It is easy to show that

$$
U_{h} \rightarrow\left(t \Sigma^{1}+(1-t) \Sigma^{2}\right) \quad \text { weak }^{*} \text { in } L^{\infty}, \quad \text { as } h \rightarrow \infty .
$$

Moreover, denoting $A_{1}^{h}=(1 / h) A_{1}$ and $A_{2}^{h}=(1 / h) A_{2}$ we have

$$
\chi_{A_{1}^{h}} \rightarrow t \quad \text { and } \quad \chi_{A_{2}^{h}} \rightarrow(1-t) \quad \text { weak }^{*} \text { in } L^{\infty}, \quad \text { as } h \rightarrow \infty .
$$

By Step 2 and the lower semicontinuity of $F$ we have

$$
\begin{aligned}
F\left(t \Sigma^{1}+(1-t) \Sigma^{2}, B_{\rho}(x)\right) & \leq \liminf _{h \rightarrow \infty} F\left(U_{h}, B_{\rho}(x)\right) \\
& =\liminf _{h \rightarrow \infty}\left(\int_{A_{1}^{h} \cap B_{\rho}(x)} \varphi\left(y, \Sigma^{1}\right) \mathrm{d} y+\int_{A_{2}^{h} \cap B_{\rho}(x)} \varphi\left(y, \Sigma^{2}\right) \mathrm{d} y\right) \\
& =t \int_{B_{\rho}(x)} \varphi\left(y, \Sigma^{1}\right) \mathrm{d} y+(1-t) \int_{B_{\rho}(x)} \varphi\left(y, \Sigma^{2}\right) \mathrm{d} y \\
& =t F\left(\Sigma^{1}, B_{\rho}(x)\right)+(1-t) F\left(\Sigma^{2}, B_{\rho}(x)\right),
\end{aligned}
$$

which implies that $\varphi$ is rank- $(n-1)$ convex in the second variable for all $x \in \Omega$. In particular, by (4.1), $\varphi$ is continuous in the second variable (see Rem. 2.3).

Step 4. Integral representation on balls.

Let $B$ be an open ball such that $\bar{B} \subset \Omega$ and let $U \in C^{\infty}\left(\Omega ; \mathbb{M}^{d \times n}\right)$ with $\operatorname{Div} U=0$ in $\Omega$. We first show that it is possible to extend the integral representation from piecewise constant functions (see Step 2) to $C^{\infty}$-functions using a suitable approximation of $U$ by piecewise constant divergence free fields. More precisely, since $B$ is a starshaped domain, by Poincaré Lemma, there exists a function $\Phi \in C^{\infty}\left(B ; \mathbb{M}^{d \times N}\right)$ with $N=n(n-1) / 2$, such that

$$
\left.U\right|_{B}=\mathcal{L} \Phi
$$

for a suitable first order linear differential operator $\mathcal{L}$ satisfying Div $\mathcal{L} \psi=0$ for every $\psi \in C^{1}\left(B ; \mathbb{M}^{d \times N}\right)$ (e.g. if $d=1$ and $n=3$ then $\mathcal{L}=\operatorname{curl}$ and $\Phi$ is the potential vector of $U$; i.e., $U=\operatorname{curl} \Phi$ ). 
Now $\Phi$ can be approximated by a sequence $\left\{\Phi_{h}\right\}$ of piecewise affine functions which converges strongly to $\Phi$ in $W^{1, p}\left(B ; \mathbb{M}^{d \times N}\right)$. Hence, $\left\{\mathcal{L} \Phi_{h}\right\}$ is a sequence of piecewise constant function with Div $\mathcal{L} \Phi_{h}=0$ and strongly converging to $\mathcal{L} \Phi=U$ in $L^{p}\left(B ; \mathbb{M}^{d \times n}\right)$. Since $F$ is lower semicontinuous, by (4.1) and Steps 2 and 3 we get

$$
F(U, B) \leq \liminf _{h \rightarrow \infty} F\left(\mathcal{L} \Phi_{h}, B\right)=\lim _{h \rightarrow \infty} \int_{B} \varphi\left(x, \mathcal{L} \Phi_{h}\right) \mathrm{d} x=\int_{B} \varphi(x, U) \mathrm{d} x .
$$

We now prove the reverse inequality. We define

$$
G(V, B)=F(U+V, B)
$$

where $U, V \in C^{\infty}\left(\Omega ; \mathbb{M}^{d \times n}\right)$ with $\operatorname{Div} U=\operatorname{Div} V=0$. Up to now we have proved that there exists a Carathéodory functions $\psi$ rank- $(n-1)$ convex in the second variable and satisfying (4.1) such that

$$
G(V, B) \leq \int_{B} \psi(x, V) \mathrm{d} x
$$

Note that, in (4.3) the equality holds if $V$ is a piecewise constant function. Hence, since $\mathcal{L} \Phi_{h} \rightarrow U$ in $L^{p}\left(B ; \mathbb{M}^{d \times n}\right)$ we have

$$
\begin{aligned}
\int_{B} \psi(x, 0) \mathrm{d} x & =G(0, B)=F(U, B) \\
& \leq \int_{B} \varphi(x, U) \mathrm{d} x=\lim _{h \rightarrow \infty} \int_{B} \varphi\left(x, \mathcal{L} \Phi_{h}\right) \mathrm{d} x \\
& =\lim _{h \rightarrow \infty} F\left(\mathcal{L} \Phi_{h}, B\right)=\lim _{h \rightarrow \infty} G\left(\mathcal{L} \Phi_{h}-U, B\right) \\
& \leq \lim _{h \rightarrow \infty} \int_{B} \psi\left(x, \mathcal{L} \Phi_{h}-U\right) \mathrm{d} x=\int_{B} \psi(x, 0) \mathrm{d} x
\end{aligned}
$$

and, in particular,

$$
F(U, B)=\int_{B} \varphi(x, U) \mathrm{d} x
$$

for all $U \in C^{\infty}\left(\Omega ; \mathbb{M}^{d \times n}\right)$, with $\operatorname{Div} U=0$ in $\Omega$.

It remains to extend the integral representation result on $B$ for $C^{\infty}$-functions to all $L^{p}$-functions by convolution. Let $U \in L^{p}\left(\Omega ; \mathbb{M}^{d \times n}\right)$ with $\operatorname{Div} U=0$ in $\Omega$ and let $\rho_{j} \in C_{c}^{\infty}\left(\mathbb{R}^{n}\right)$ such that $\int_{\mathbb{R}^{n}} \rho_{j} \mathrm{~d} x=1$ and $\operatorname{spt} \rho_{j} \subset B(0,1 / j)$ where $1 / j<\operatorname{dist}\left(B, \mathbb{R}^{n} \backslash \Omega\right)$. Then $\rho_{j} * U \in C^{\infty}\left(B ; \mathbb{M}^{d \times n}\right)$, with $\operatorname{Div}\left(\rho_{j} * U\right)=0$ in $B$, and

$$
U_{j}:=\rho_{j} * U \rightarrow U \quad \text { in } \quad L^{p}\left(B ; \mathbb{M}^{d \times n}\right) .
$$

By (4.4) we have

$$
F(U, B) \leq \liminf _{j \rightarrow \infty} F\left(U_{j}, B\right)=\lim _{j \rightarrow \infty} \int_{B} \varphi\left(x, U_{j}\right) \mathrm{d} x=\int_{B} \varphi(x, U) \mathrm{d} x .
$$

The reverse inequality follows using the previous argument with $\left\{\rho_{j} * U\right\}$ in place of $\left\{\mathcal{L} \Phi_{h}\right\}$.

Step 5. Integral representation on $A \in \mathcal{A}(\Omega)$.

Let $A \in \mathcal{A}(\Omega)$, by the Vitali Covering Theorem, there exists a disjoint sequence $\left\{\bar{B}_{j}\right\}$ of closed balls, subsets of $A$, such that $\left|A \backslash \bigcup_{j} \bar{B}_{j}\right|=0$. Since $F$ is a Borel measure absolutely continuous with respect to the Lesbegue 
measure, by Step 4 we have that

$$
F(U, A)=\sum_{j} F\left(U, B_{j}\right)=\sum_{j} \int_{B_{j}} \varphi(x, U) \mathrm{d} x=\int_{A} \varphi(x, U) \mathrm{d} x
$$

which gives the integral representation of $F$ for every open set $A \in \mathcal{A}(\Omega)$.

Finally, by the lower-semicontinuity of $F$, we have that $\varphi$ is also Div-quasiconvex which concludes the proof.

\section{BOUNDARY CONDITIONS AND VOLUME CONSTRAINTS}

In this section we study the stability of the $\Gamma$-convergence result, obtained in Section 4, under average, periodicity and boundary conditions. The main tool is again the fundamental estimate that allows us to modify the optimal sequence for the $\Gamma$-limit in a new sequence that matches additional conditions. This study is also motivated by the possible application of our general $\Gamma$-convergence result to the case of homogenization (see Sect. 6).

We recall that

$$
L_{\#, \text { Div }}^{p}\left(Q ; \mathbb{M}^{d \times n}\right)=\left\{U \in L_{\text {loc }}^{p}\left(\mathbb{R}^{n} ; \mathbb{M}^{d \times n}\right): Q \text {-periodic, Div } U=0 \text { in } \mathbb{R}^{n}\right\},
$$

where $Q$ denotes the unit cube in $\mathbb{R}^{n}$.

Proposition $\mathbf{5 . 1}$ (average and periodicity condition). Let $\left\{F_{j}\right\}$ be a sequence of functionals defined by (2.10) and satisfying the conditions (2.5), (2.6), such that

$$
F(U, Q)=\Gamma-\lim _{j \rightarrow+\infty} F_{j}(U, Q)
$$

for all $U \in L^{p}\left(Q ; \mathbb{M}^{d \times n}\right)$ with $\operatorname{Div} U=0$ in $Q$. Then, the sequence of functionals

$$
G_{j}(U, Q)= \begin{cases}F_{j}(U, Q) & U \in L_{\#, \text { Div }}^{p}\left(Q ; \mathbb{M}^{d \times n}\right), f_{Q} U \mathrm{~d} x=m \\ +\infty & \text { otherwise }\end{cases}
$$

$\Gamma$-converges to

$$
G(U, Q)= \begin{cases}F(U, Q) & U \in L_{\#, \text { Div }}^{p}\left(Q ; \mathbb{M}^{d \times n}\right), f_{Q} U \mathrm{~d} x=m \\ +\infty & \text { otherwise }\end{cases}
$$

with $m \in \mathbb{M}^{d \times n}$.

Proof. Let $U \in L_{\# \text {,Div }}^{p}\left(Q ; \mathbb{M}^{d \times n}\right)$, with $f_{Q} U \mathrm{~d} x=m$. By the $\Gamma$-convergence of $\left\{F_{j}(\cdot, Q)\right\}$ to $F(\cdot, Q)$, there exists a sequence $\left\{U_{j}\right\}$ weakly converging to $U$ in $L^{p}\left(Q ; \mathbb{M}^{d \times n}\right)$, with $\operatorname{Div} U_{j}=0$, such that

$$
G(U, Q)=F(U, Q)=\lim _{j \rightarrow \infty} F_{j}\left(U_{j}, Q\right) .
$$

Let $K$ be a compact set and $A^{\prime} \in \mathcal{A}(Q)$ such that $K \subset A^{\prime} \subset \subset Q$. By Remark 3.3 with $V_{j}=U, A=Q$ and $B=Q \backslash K$, there exists a sequence $\left\{W_{j}\right\}$, defined as in (3.10), such that

$$
\left\{\begin{array}{l}
W_{j} \rightarrow U \quad \text { in } \quad L^{p}\left(Q ; \mathbb{M}^{d \times n}\right) \\
W_{j} \in L_{\#, \text { Div }}^{p}\left(Q ; \mathbb{M}^{d \times n}\right), \quad f_{Q} W_{j} \mathrm{~d} x=f_{Q} U \mathrm{~d} x=m
\end{array}\right.
$$


and

$$
F_{j}\left(W_{j}, Q\right) \leq F_{j}\left(U_{j}, Q\right)+F_{j}(U, Q \backslash K)+\sigma+o(1)
$$

as $j \rightarrow+\infty$. Note that, the periodicity of the sequence $\left\{W_{j}\right\}$ follows from the periodicity assumption on $U$. By (2.5), we can choose $K$ such that $F_{j}(U, Q \backslash K) \leq \sigma$. Then

$$
G_{j}\left(W_{j}, Q\right)=F_{j}\left(W_{j}, Q\right) \leq F_{j}\left(U_{j}, Q\right)+2 \sigma+o(1),
$$

and hence

$$
\limsup _{j \rightarrow \infty} G_{j}\left(W_{j}, Q\right) \leq \lim _{j \rightarrow \infty} F_{j}\left(U_{j}, Q\right)+2 \sigma=G(U, Q)+2 \sigma .
$$

By the arbitrariness of $\sigma$ we get the limsup inequality; i.e.,

$$
\Gamma-\limsup _{j \rightarrow+\infty} G_{j}(U, Q) \leq G(U, Q) .
$$

The lim inf inequality is trivially satisfied since

$$
\Gamma-\liminf _{j \rightarrow+\infty} G_{j}(U, Q) \geq \Gamma-\liminf _{j \rightarrow+\infty} F_{j}(U, Q)
$$

for every $U \in L_{\#, \text { Div }}^{p}\left(Q ; \mathbb{M}^{d \times n}\right)$ with $f_{Q} U \mathrm{~d} x=m$.

Remark 5.2 (average conditions). Clearly, reasoning as in Proposition 5.1, we can also prove that given $\left\{F_{j}\right\}$ as above, the sequence of functionals

$$
G_{j}(U, A)= \begin{cases}F_{j}(U, A) & U \in L^{p}\left(A ; \mathbb{M}^{d \times n}\right), \operatorname{Div} U=0, f_{A} U \mathrm{~d} x=m \\ +\infty & \text { otherwise }\end{cases}
$$

$\Gamma$-converges to

$$
G(U, A)= \begin{cases}F(U, A) & U \in L^{p}\left(A ; \mathbb{M}^{d \times n}\right), \operatorname{Div} U=0, f_{A} U \mathrm{~d} x=m \\ +\infty & \text { otherwise }\end{cases}
$$

with $m \in \mathbb{M}^{d \times n}$ and $A \in \mathcal{A}(\Omega)$.

We conclude this section by considering a natural boundary condition for this problem. Recall that for every $U \in L^{p}\left(\Omega ; \mathbb{M}^{d \times n}\right)$, satisfying $\operatorname{Div} U=0$ in $\Omega$, it is possible to define the normal trace $U \cdot \nu$ on $\partial \Omega$, whenever $\partial \Omega$ is Lipschitz regular. In particular, the trace belongs to the space $W^{-\frac{1}{p}, p}\left(\partial \Omega, \mathbb{R}^{d}\right)$ (see e.g. [2] and [15]).

Proposition 5.3 (boundary condition). Let $\left\{F_{j}\right\}$ be a sequence of functionals defined by (2.10) and satisfying the conditions (2.5), (2.6) such that

$$
F(U, A)=\Gamma-\lim _{j \rightarrow+\infty} F_{j}(U, A)
$$

for all $U \in L^{p}\left(A ; \mathbb{M}^{d \times n}\right)$ with $\operatorname{Div} U=0$ in $A$ and $A \in \mathcal{A}(\Omega)$ with Lipschitz boundary. Then, for every $g \in W^{-\frac{1}{p}, p}\left(\partial \Omega, \mathbb{R}^{d}\right)$ the sequence of functionals

$$
G_{j}(U, A)= \begin{cases}F_{j}(U, A) & U \in L^{p}\left(A ; \mathbb{M}^{d \times n}\right), \operatorname{Div} U=0, U \cdot \nu=g \text { on } \partial A \\ +\infty & \text { otherwise }\end{cases}
$$


$\Gamma$-converges to

$$
G(U, A)= \begin{cases}F(U, A) & U \in L^{p}\left(A ; \mathbb{M}^{d \times n}\right), \operatorname{Div} U=0, U \cdot \nu=g \text { on } \partial A \\ +\infty & \text { otherwise }\end{cases}
$$

where $\nu$ is the outer normal on $\partial A$.

In particular, for every $U \in L^{p}\left(A ; \mathbb{M}^{d \times n}\right)$, with $\operatorname{Div} U=0$, there exists a sequence $\left\{W_{j}\right\} \subseteq L^{p}\left(\mathbb{R}^{n} ; \mathbb{M}^{d \times n}\right)$ weakly converging to 0 in $L^{p}\left(\mathbb{R}^{n} ; \mathbb{M}^{d \times n}\right)$ such that $\operatorname{Div} W_{j}=0$ in $\mathbb{R}^{n}, W_{j}=0$ in $\mathbb{R}^{n} \backslash A, \int_{A} W_{j} \mathrm{~d} x=0$ and

$$
F(U, A)=\lim _{j \rightarrow+\infty} F_{j}\left(U+W_{j}, A\right) .
$$

Proof. Let $U \in L^{p}\left(A ; \mathbb{M}^{d \times n}\right)$ with $\operatorname{Div} U=0$ on $A$ and $U \cdot \nu=g$ on $\partial A$. By assumption there exists a sequence $\left\{U_{j}\right\} \subseteq L^{p}\left(A ; \mathbb{M}^{d \times n}\right)$ weakly converging to $U$ in $L^{p}\left(A ; \mathbb{M}^{d \times n}\right)$, with $\operatorname{Div} U_{j}=0$, such that

$$
G(U, A)=F(U, A)=\lim _{j \rightarrow \infty} F_{j}\left(U_{j}, A\right)
$$

Let $K$ be a compact set and $A^{\prime} \in \mathcal{A}(\Omega)$ such that $K \subset A^{\prime} \subset \subset A$. Reasoning as in the proof of the fundamental estimate (with $V_{j}=U$ and $B=A \backslash K$ ) we can show that for every $\sigma>0$ there exists a sequence $\left\{\widetilde{W}_{j}\right\}$ such that

as $j \rightarrow+\infty$, where

$$
F_{j}\left(\widetilde{W}_{j}, A\right) \leq F_{j}\left(U_{j}, A\right)+F_{j}(U, A \backslash K)+\sigma+o(1)
$$

$$
\left\{\begin{array}{l}
\left(\widetilde{W}_{j}\right)_{i}=\varphi_{j}\left(U_{j}\right)_{i}+\left(1-\varphi_{j}\right)(U)_{i}+\left|D\left(g_{j}\right)_{i}\right|^{p^{\prime}-2} D\left(g_{j}\right)_{i} \quad i=1, \ldots, d \\
\widetilde{W}_{j} \rightarrow U \text { in } \quad L^{p}\left(A ; \mathbb{M}^{d \times n}\right) \\
\widetilde{W}_{j} \cdot \nu=U \cdot \nu \quad \text { on } \partial A \\
\operatorname{Div} \widetilde{W}_{j}=0 \quad \text { on } A
\end{array}\right.
$$

$\varphi_{j} \in C_{0}^{\infty}(A)$ is a suitable cut-off function between $A^{\prime}$ and $A$ and $g_{j}$ is the solution of the Neumann system

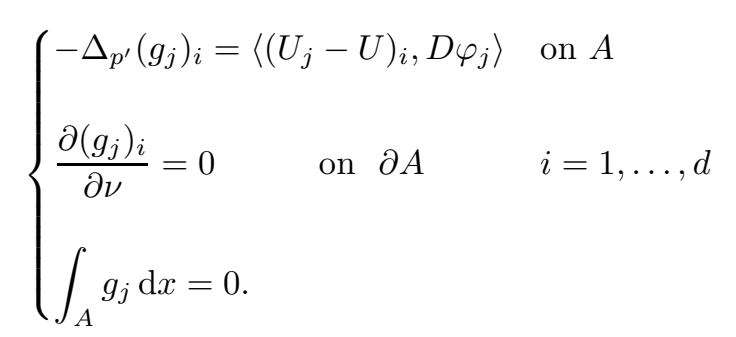

Note that if a vector field $v: A \rightarrow \mathbb{R}^{n}$ satisfies $\operatorname{div} v=0$ in $A$ and $\langle v, \nu\rangle=0$ on $\partial A$, then $\int_{A} v \mathrm{~d} x=0$. In fact, applying the divergence theorem to the function $(x)_{i} v$, where $(x)_{i}$ is the $i$ th component of $x$ for every $i=1, \ldots, n$, we get

$$
\int_{A}(v)_{i} \mathrm{~d} x=\int_{A} \operatorname{div}\left((x)_{i} v\right) \mathrm{d} x=\int_{\partial A}(x)_{i}\langle v, \nu\rangle \mathrm{d} \mathcal{H}^{n-1}=0 .
$$

Then, as a consequence of $(5.5)$, we have that

$$
\int_{A} \widetilde{W}_{j} \mathrm{~d} x=\int_{A} U \mathrm{~d} x
$$

By (2.5), we can choose $K$ such that $F_{j}(U, A \backslash K) \leq \sigma$ and hence

$$
G_{j}\left(\widetilde{W}_{j}, A\right)=F_{j}\left(\widetilde{W}_{j}, A\right) \leq F_{j}\left(U_{j}, A\right)+2 \sigma+o(1)
$$


By the arbitrariness of $\sigma$, we get the limsup inequality

$$
\Gamma-\limsup _{j \rightarrow \infty} G_{j}(U, A) \leq G(U, A) .
$$

The lim inf inequality is trivially satisfied since

$$
\Gamma-\liminf _{j \rightarrow+\infty} G_{j}(U, Q) \geq \Gamma-\liminf _{j \rightarrow+\infty} F_{j}(U, Q)
$$

hence, we get the $\Gamma$-convergence result.

Note that, in particular, we have proved that for every $U \in L^{p}\left(A ; \mathbb{M}^{d \times n}\right)$, with $\operatorname{Div} U=0$ in $A$, there exists a sequence $\left\{\widetilde{W}_{j}\right\}$ as in (5.5) such that

$$
\lim _{j \rightarrow+\infty} F_{j}\left(\widetilde{W}_{j}, A\right)=F(U, A)
$$

We now define $W_{j}=\widetilde{W}_{j}-U$. Since $W_{j} \cdot \nu=0$ on $\partial A$ we can extend it equal to zero outside $A$ keeping the divergence constraint in $\mathbb{R}^{n}$. Hence, we can rewrite

$$
\lim _{j \rightarrow+\infty} F_{j}\left(U+W_{j}, A\right)=F(U, A),
$$

where, by (5.5) and (5.6), $\left\{W_{j}\right\} \subseteq L^{p}\left(\mathbb{R}^{n} ; \mathbb{M}^{d \times n}\right)$ satisfies the following properties

$$
\left\{\begin{array}{l}
W_{j} \rightarrow 0 \quad \text { in } \quad L^{p}\left(\mathbb{R}^{n} ; \mathbb{M}^{d \times n}\right) \\
\operatorname{Div} W_{j}=0 \quad \text { in } \quad \mathbb{R}^{n} \\
W_{j}=0 \quad \text { in } \quad \mathbb{R}^{n} \backslash A, \quad \int_{A} W_{j} \mathrm{~d} x=0 .
\end{array}\right.
$$

\section{Homogenization}

We can apply our general $\Gamma$-convergence result to the case of homogenization. In this section we propose an alternative proof to the one due to Braides, Fonseca and Leoni in [5] of the homogenization formula describing the integrand function $\varphi$.

Theorem 6.1. Let $f: \mathbb{R}^{n} \times \mathbb{M}^{d \times n} \mapsto[0,+\infty)$ be a Borel function, Q-periodic in the first variable, satisfying (2.5) and (2.6) with $f$ in place of $f_{j}$. Let us consider the family of functionals $\left\{F_{\varepsilon}\right\}$ defined by

$$
F_{\varepsilon}(U, \Omega)= \begin{cases}\int_{\Omega} f\left(\frac{x}{\varepsilon}, U\right) \mathrm{d} x & U \in L^{p}\left(\Omega ; \mathbb{M}^{d \times n}\right), \operatorname{Div} U=0 \text { in } \Omega \\ +\infty & \text { otherwise. }\end{cases}
$$

Then, we have

$$
\Gamma-\lim _{\varepsilon \rightarrow 0} F_{\varepsilon}(U, \Omega)=\int_{\Omega} f_{\mathrm{hom}}(U) \mathrm{d} x,
$$

for every $U \in L^{p}\left(\Omega ; \mathbb{M}^{d \times n}\right)$ with $\operatorname{Div} U=0$ in $\Omega$, where $f_{\mathrm{hom}}: \mathbb{M}^{d \times n} \mapsto[0,+\infty)$ is a Div -quasiconvex function described by the following homogenization formula

$$
f_{\text {hom }}(\Sigma)=\lim _{t \rightarrow+\infty} \frac{1}{t^{n}} \inf \left\{\int_{t Q} f(y, \Sigma+V(y)) \mathrm{d} y: V \in L_{\#, \text { Div }}^{p}\left(t Q ; \mathbb{M}^{d \times n}\right), \int_{t Q} V \mathrm{~d} y=0\right\}
$$

for every $\Sigma \in \mathbb{M}^{d \times n}$ with $Q=(-1 / 2,1 / 2)^{n}$. 
Moreover, if $d=1$ then $f_{\mathrm{hom}}$ is convex and it is given by the cell-problem formula

$$
f_{\text {hom }}(\xi)=\inf \left\{\int_{Q} f(y, \xi+v(y)) \mathrm{d} y: v \in L_{\#, \text { div }}^{p}\left(Q ; \mathbb{R}^{n}\right), \int_{Q} v \mathrm{~d} y=0\right\}
$$

for every $\xi \in \mathbb{R}^{n}$.

Proof. We recall that, by Theorem 4.2, for every sequence $\left\{\varepsilon_{j}\right\}$ converging to 0 there exist a subsequence of $\left\{\varepsilon_{j}\right\}$ (not relabeled) and a function $\varphi: \Omega \times \mathbb{M}^{d \times n} \mapsto[0,+\infty)$, Div-quasiconvex in the second variable, such that

$$
\Gamma-\lim _{j \rightarrow+\infty} F_{\varepsilon_{j}}(U, A)=\int_{A} \varphi(x, U) \mathrm{d} x
$$

for all $U \in L^{p}\left(\Omega ; \mathbb{M}^{d \times n}\right)$ with $\operatorname{Div} U=0$ in $\Omega$ and $A \in \mathcal{A}(\Omega)$.

Step 1. $\varphi$ does not depend on $x$.

It is sufficient to prove that if $\Sigma \in \mathbb{M}^{d \times n}, y, z \in \mathbb{R}^{n}$ and $\rho>0$ then

$$
\Gamma-\lim _{j \rightarrow+\infty} F_{\varepsilon_{j}}\left(\Sigma, B_{\rho}(y)\right)=\Gamma-\lim _{j \rightarrow+\infty} F_{\varepsilon_{j}}\left(\Sigma, B_{\rho}(z)\right) .
$$

The proof that follows is a classical argument in homogenization. We briefly repeat it for the reader convenience (see e.g. [4], Prop. 14.3). By Proposition 5.3 there exists a sequence $U_{j} \rightarrow 0$ in $L^{p}\left(\mathbb{R}^{n} ; \mathbb{M}^{d \times n}\right)$, with $\operatorname{Div} U_{j}=0$ in $\mathbb{R}^{n}$ and $U_{j}=0$ in $\mathbb{R}^{n} \backslash B_{\rho}(y)$, such that

$$
\Gamma-\lim _{j \rightarrow+\infty} F_{\varepsilon_{j}}\left(\Sigma, B_{\rho}(y)\right)=\lim _{j \rightarrow \infty} F_{\varepsilon_{j}}\left(\Sigma+U_{j}, B_{\rho}(y)\right) .
$$

Let

for $i=1, \ldots, n$, then

$$
\left(\tau_{j}\right)_{i}=\varepsilon_{j}\left[\frac{z_{i}-y_{i}}{\varepsilon_{j}}\right], \quad V_{j}(x)=U_{j}\left(x-\tau_{j}\right)
$$

$$
F_{\varepsilon_{j}}\left(\Sigma+V_{j}, \tau_{j}+B_{\rho}(y)\right)=F_{\varepsilon_{j}}\left(\Sigma+U_{j}, B_{\rho}(y)\right) .
$$

Moreover, $V_{j}=0$ in $\mathbb{R}^{n} \backslash\left(\tau_{j}+B_{\rho}(y)\right)$, with $\operatorname{Div} V_{j}=0$ and $V_{j} \rightarrow 0$ in $L^{p}\left(B_{r \rho}(z) ; \mathbb{M}^{d \times n}\right)$ for $r>1$. Hence,

$$
\begin{aligned}
\Gamma-\lim _{j \rightarrow+\infty} F_{\varepsilon_{j}}\left(\Sigma, B_{\rho}(z)\right) & \leq \Gamma-\lim _{j \rightarrow+\infty} F_{\varepsilon_{j}}\left(\Sigma, B_{r \rho}(z)\right) \\
& \leq \liminf _{j \rightarrow+\infty} F_{\varepsilon_{j}}\left(\Sigma+V_{j}, B_{r \rho}(z)\right) \leq \liminf _{j \rightarrow+\infty} F_{\varepsilon_{j}}\left(\Sigma+U_{j}, B_{\rho}(y)\right)+\beta\left(1+\|\Sigma\|^{p}\right)\left|B_{r \rho} \backslash B_{\rho}\right| \\
& =\Gamma-\lim _{j \rightarrow+\infty} F_{\varepsilon_{j}}\left(\Sigma, B_{\rho}(y)\right)+\beta\left(1+\|\Sigma\|^{p}\right)\left|B_{r \rho} \backslash B_{\rho}\right| .
\end{aligned}
$$

Passing to the limit as $r \rightarrow 1$ we have that

$$
\Gamma-\lim _{j \rightarrow+\infty} F_{\varepsilon_{j}}\left(\Sigma, B_{\rho}(z)\right) \leq \Gamma-\lim _{j \rightarrow+\infty} F_{\varepsilon_{j}}\left(\Sigma, B_{\rho}(y)\right) .
$$

By symmetry we obtain the reverse inequality.

Step 2. Asymptotic formula.

We first prove that the following limit exists

$$
\lim _{t \rightarrow+\infty} \inf \left\{\frac{1}{t^{n}} \int_{t Q} f(y, \Sigma+V(y)) \mathrm{d} y: V \in L^{p}\left(t Q ; \mathbb{M}^{d \times n}\right), \operatorname{Div} V=0, V \cdot \nu=0 \text { on } \partial(t Q)\right\} .
$$


Fixed $\Sigma \in \mathbb{M}^{d \times n}$, for $t>0$ we define

$$
g_{t}=\frac{1}{t^{n}} \inf \left\{\int_{t Q} f(y, \Sigma+V(y)) \mathrm{d} y: V \in L^{p}\left(t Q ; \mathbb{M}^{d \times n}\right), \operatorname{Div} V=0, V \cdot \nu=0 \text { on } \partial(t Q)\right\}
$$

hence, there exists $V_{t} \in L^{p}\left(t Q ; \mathbb{M}^{d \times n}\right)$, with $\operatorname{Div} V_{t}=0$ and $V_{t} \cdot \nu=0$ on $\partial(t Q)$, such that

$$
\frac{1}{t^{n}} \int_{t Q} f\left(y, \Sigma+V_{t}(y)\right) \mathrm{d} y \leq g_{t}+\frac{1}{t}
$$

Let $s>t$, we denote by $I=\left\{i \in \mathbb{Z}^{n}: 0 \leq([t]+1)\left|(i)_{j}\right|<(s / 2), j=1, \ldots, n\right\}$ and we define the function

$$
V_{s}(x)= \begin{cases}V_{t}(x-i) & \text { if } x \in i+t Q, i \in I \\ 0 & \text { otherwise }\end{cases}
$$

and the set $Q_{s}=s Q \backslash \bigcup_{i \in I}(i+t Q)$. Note that $V_{s} \in L^{p}\left(s Q ; \mathbb{M}^{d \times n}\right)$, Div $V_{s}=0$ and $V_{s} \cdot \nu=0$ on $\partial(s Q)$; moreover,

$$
\begin{aligned}
g_{s} & \leq \frac{1}{s^{n}} \int_{s Q} f\left(x, \Sigma+V_{s}(x)\right) \mathrm{d} x=\frac{1}{s^{n}}\left(\sum_{i \in I} \int_{t Q+i} f\left(x, \Sigma+V_{t}(x-i)\right) \mathrm{d} x+\int_{Q_{s}} f(x, \Sigma) \mathrm{d} x\right) \\
& \leq \frac{1}{s^{n}}\left(\sum_{i \in I} \int_{t Q} f\left(y, \Sigma+V_{t}(y)\right) \mathrm{d} y+c\left|Q_{s}\right|\right) \leq \frac{t^{n}}{([t]+1)^{n}}\left(g_{t}+\frac{1}{t}\right)+c \frac{\left|Q_{s}\right|}{s^{n}},
\end{aligned}
$$

where $\left|Q_{s}\right|$ denotes the Lesbegue measure of $Q_{s}$. Taking first the limsup as $s \rightarrow+\infty$ and then the lim inf as $t \rightarrow+\infty$ we get

$$
\limsup _{s \rightarrow+\infty} g_{s} \leq \liminf _{t \rightarrow+\infty} g_{t}
$$

which concludes the proof of the existence of the limit in (6.8).

Since $\varphi$ is Div-quasiconvex we have

$$
\begin{aligned}
\varphi(\Sigma) & =\inf \left\{\int_{Q} \varphi(\Sigma+V(y)) \mathrm{d} y: V \in L_{\#, \text { Div }}^{p}\left(Q ; \mathbb{M}^{d \times n}\right), \int_{Q} V \mathrm{~d} y=0\right\} \\
& \leq \inf \left\{\int_{Q} \varphi(\Sigma+V(y)) \mathrm{d} y: V \in L^{p}\left(Q ; \mathbb{M}^{d \times n}\right), \operatorname{Div} V=0, V \cdot \nu=0 \text { on } \partial Q\right\} \\
& \leq \varphi(\Sigma)
\end{aligned}
$$

where the last inequality is obtained choosing as test function $V=0$. Hence, by (6.7), Proposition 5.3, (6.8) and the convergence of the minimum problems assured by the $\Gamma$-convergence, we have that

$$
\begin{aligned}
\varphi(\Sigma) & =\inf \left\{\int_{Q} \varphi(\Sigma+V(y)) \mathrm{d} y: V \in L^{p}\left(Q ; \mathbb{M}^{d \times n}\right), \operatorname{Div} V=0, V \cdot \nu=0 \text { on } \partial Q\right\} \\
& =\lim _{j \rightarrow \infty} \inf \left\{\int_{Q} f\left(\frac{x}{\varepsilon_{j}}, \Sigma+V(x)\right) \mathrm{d} x: V \in L^{p}\left(Q ; \mathbb{M}^{d \times n}\right), \operatorname{Div} V=0, V \cdot \nu=0 \text { on } \partial Q\right\} \\
& =\lim _{t \rightarrow+\infty} \frac{1}{t^{n}} \inf \left\{\int_{t Q} f(y, \Sigma+V(y)) \mathrm{d} y: V \in L^{p}\left(t Q ; \mathbb{M}^{d \times n}\right), \operatorname{Div} V=0, V \cdot \nu=0 \text { on } \partial(t Q)\right\}
\end{aligned}
$$


Step 3. $\Gamma$-convergence of the whole family $\left\{F_{\varepsilon}\right\}$.

From Step 2 we can, in particular, deduce that $\varphi$ does not depend on $\left\{\varepsilon_{j}\right\}$; hence, the whole family $\left\{F_{\varepsilon}\right\}$ $\Gamma$-converges; i.e.,

$$
\Gamma-\lim _{\varepsilon \rightarrow 0} F_{\varepsilon}(U, A)=\int_{A} \varphi(U) \mathrm{d} x
$$

for all $U \in L^{p}\left(\Omega ; \mathbb{M}^{d \times n}\right)$, with $\operatorname{Div} U=0$ in $\Omega$, and $A \in \mathcal{A}(\Omega)$.

Step 4. Homogenization formula.

We finally prove that $\varphi$ coincides with

$$
f_{\text {hom }}(\Sigma):=\lim _{t \rightarrow+\infty} \frac{1}{t^{n}} \inf \left\{\int_{t Q} f(y, \Sigma+V(y)) \mathrm{d} y: V \in L_{\#, \text { Div }}^{p}\left(t Q ; \mathbb{M}^{d \times n}\right), \int_{t Q} V \mathrm{~d} y=0\right\}
$$

for every $\Sigma \in \mathbb{M}^{d \times n}$, with $Q=(-1 / 2,1 / 2)^{n}$. By Step 2 the inequality $\varphi(\Sigma) \geq f_{\text {hom }}(\Sigma)$ is trivial. We then deal with the reverse inequality.

For every $\Sigma \in \mathbb{M}^{d \times n}$ we define

$$
f_{\text {hom }}^{t}(\Sigma)=\frac{1}{t^{n}} \inf \left\{\int_{t Q} f(y, \Sigma+V(y)) \mathrm{d} y: V \in L_{\#, \text { Div }}^{p}\left(t Q ; \mathbb{M}^{d \times n}\right), \int_{t Q} V \mathrm{~d} y=0\right\} .
$$

For $t>0$, let $V_{t} \in L_{\#, \text { Div }}^{p}\left(t Q ; \mathbb{M}^{d \times n}\right)$ with $\int_{t Q} V_{t} \mathrm{~d} y=0$ such that

$$
\frac{1}{t^{n}} \int_{t Q} f\left(y, \Sigma+V_{t}(y)\right) \mathrm{d} y \leq f_{\text {hom }}^{t}(\Sigma)+\frac{1}{t}
$$

We extend $V_{t}$ by periodicity to the whole $\mathbb{R}^{n}$ and define $V_{j}(x):=V_{t}\left(x / \varepsilon_{j}\right)$. Then $V_{j} \rightarrow 0$ in $L^{p}\left(Q ; \mathbb{M}^{d \times n}\right)$, as $j \rightarrow+\infty$, and $\operatorname{Div} V_{j}=0$. Moreover, by Step 3 we may fix $\varepsilon_{j}=1 / j t$; hence,

$$
\begin{aligned}
\varphi(\Sigma) & \leq \liminf _{j \rightarrow+\infty} \int_{Q} f\left(\frac{x}{\varepsilon_{j}}, \Sigma+V_{j}(x)\right) \mathrm{d} x=\liminf _{j \rightarrow+\infty} \frac{1}{(j t)^{n}} \int_{j t Q} f\left(y, \Sigma+V_{t}(y)\right) \mathrm{d} y \\
& =\frac{1}{t^{n}} \int_{t Q} f\left(y, \Sigma+V_{t}(y)\right) \mathrm{d} y \leq f_{\text {hom }}^{t}(\Sigma)+\frac{1}{t}
\end{aligned}
$$

Passing to the limit as $t$ tends to $+\infty$ we get the reverse inequality which concludes the proof of the homogenization formula.

Step 5. Homogenization formula for $d=1$.

If $d=1$, then $\varphi$ coincides with

$$
f_{\text {hom }}(\xi):=\inf \left\{\int_{Q} f(y, \xi+v(y)) \mathrm{d} y: v \in L_{\#, \text { div }}^{p}\left(Q ; \mathbb{R}^{n}\right), \int_{Q} v \mathrm{~d} y=0\right\}
$$

for every $\xi \in \mathbb{R}^{n}$.

In fact, by Step 4 we have that

$$
\varphi(\xi)=\lim _{t \rightarrow+\infty} \frac{1}{t^{n}} \inf \left\{\int_{t Q} f(y, \xi+v(y)) \mathrm{d} y: v \in L_{\#, \text { div }}^{p}\left(t Q ; \mathbb{R}^{n}\right), \int_{t Q} v \mathrm{~d} y=0\right\}
$$

hence, the inequality $\varphi(\xi) \leq f_{\text {hom }}(\xi)$ is trivial. 
We fix $\varepsilon_{j}=1 / j$. By Step 3 and Proposition 5.1 there exists a sequence $\left\{u_{j}\right\} \subseteq L_{\# \text {,div }}^{p}\left(Q ; \mathbb{R}^{n}\right)$ weakly converging to 0 in $L^{p}\left(Q ; \mathbb{R}^{n}\right)$ with $\operatorname{div} u_{j}=0$ and $\int_{Q} u_{j} \mathrm{~d} x=0$ such that

$$
\varphi(\xi)=\lim _{j \rightarrow+\infty} \int_{Q} f\left(j x, \xi+u_{j}\right) \mathrm{d} x .
$$

By Remark 2.1 and Proposition 5.1 we have that

$$
\begin{aligned}
& \inf \left\{\int_{j Q} f^{* *}(y, \xi+v(y)) \mathrm{d} y: v \in L_{\#, \text { div }}^{p}\left(j Q ; \mathbb{R}^{n}\right), \int_{j Q} v \mathrm{~d} y=0\right\} \\
= & \inf \left\{\int_{j Q} f(y, \xi+v(y)) \mathrm{d} y: v \in L_{\#, \text { div }}^{p}\left(j Q ; \mathbb{R}^{n}\right), \int_{j Q} v \mathrm{~d} y=0\right\}
\end{aligned}
$$

for every $j \in \mathbb{N}$. Hence, by (6.9), we have that

$$
\begin{aligned}
\varphi(\xi) & =\lim _{j \rightarrow \infty} \frac{1}{j^{n}} \inf \left\{\int_{j Q} f(y, \xi+v(y)) \mathrm{d} y: v \in L_{\#, \text { div }}^{p}\left(j Q ; \mathbb{R}^{n}\right), \int_{j Q} v \mathrm{~d} y=0\right\} \\
& =\lim _{j \rightarrow \infty} \frac{1}{j^{n}} \inf \left\{\int_{j Q} f^{* *}(y, \xi+v(y)) \mathrm{d} y: v \in L_{\#, \text { div }}^{p}\left(j Q ; \mathbb{R}^{n}\right), \int_{j Q} v \mathrm{~d} y=0\right\} .
\end{aligned}
$$

Fix $\eta>0$ and consider $v_{j} \in L_{\#, \text { div }}^{p}\left(j Q ; \mathbb{R}^{n}\right)$, with $\int_{j Q} v_{j} \mathrm{~d} y=0$, such that

$$
\int_{j Q} f^{* *}\left(y, \xi+v_{j}(y)\right) \mathrm{d} y \leq \inf \left\{\int_{j Q} f^{* *}(y, \xi+v(y)) \mathrm{d} y: v \in L_{\#, \operatorname{div}}^{p}\left(j Q ; \mathbb{R}^{n}\right), \int_{j Q} v \mathrm{~d} y=0\right\}+\eta .
$$

We now define the convex combination

$$
v_{j}^{\sharp}(y)=\frac{1}{j^{n}} \sum_{i \in I_{j}} v_{j}(y+i)
$$

where $I_{j}=\{0, \ldots,(j-1)\}^{n}$. The sequence $\left\{v_{j}^{\sharp}\right\}$ is $Q$-periodic with $\operatorname{div} v_{j}^{\sharp}=0$ and $\int_{Q} v_{j}^{\sharp} \mathrm{d} y=0$. Then, we get

$$
\begin{aligned}
& \int_{j Q} f^{* *}\left(y, \xi+v_{j}(y)\right) \mathrm{d} y=\sum_{i \in I_{j}} \frac{1}{j^{n}} \int_{j Q} f^{* *}\left(y, \xi+v_{j}(y+i)\right) \mathrm{d} y \\
\geq & \int_{j Q} f^{* *}\left(y, \xi+v_{j}^{\sharp}(y)\right) \mathrm{d} y=j^{n} \int_{Q} f^{* *}\left(y, \xi+v_{j}^{\sharp}(y)\right) \mathrm{d} y \\
\geq & j^{n} \inf \left\{\int_{Q} f^{* *}(y, \xi+v(y)) \mathrm{d} y: v \in L_{\#, \operatorname{div}}^{p}\left(Q ; \mathbb{R}^{n}\right), \int_{Q} v \mathrm{~d} y=0\right\} \\
= & j^{n} f_{\text {hom }}(\xi) .
\end{aligned}
$$

By (6.10), (6.11) and by the arbitrariness of $\eta$, passing to the limit as $j \rightarrow+\infty$, we prove that $\varphi(\xi) \geq f_{\text {hom }}(\xi)$ which concludes the proof of the homogenization formula for $d=1$. of Rome "La Sapienza", where most of this work was carried out and supported. 


\section{REFERENCES}

[1] E. Acerbi and N. Fusco, Semicontinuity problems in the calculus of variations. Arch. Rational Mech. Anal. 86 (1984) 125-145.

[2] R.A. Adams, Sobolev spaces. Academic Press, New York (1975).

[3] A. Braides, $\Gamma$-convergence for Beginners. Oxford University Press, Oxford (2002).

[4] A. Braides and A. Defranceschi, Homogenization of Multiple Integrals. Oxford University Press, Oxford (1998).

[5] A. Braides, I. Fonseca and G. Leoni, A-Quasiconvexity: Relaxation and Homogenization. ESAIM: COCV 5 (2000) 539-577.

[6] G. Dal Maso, An Introduction to $\Gamma$-convergence. Birkhäuser, Boston (1993).

[7] I. Fonseca and S. Müller, A-Quasiconvexity, lower semicontinuity and Young measures. SIAM J. Math. Anal. 30 (1999) $1355-1390$.

[8] I. Fonseca, S. Müller and P. Pedregal, Analysis of concentration and oscillation effects generated by gradient. SIAM J. Math. Anal. 29 (1998) 736-756.

[9] F. Murat, Compacité par compensation : condition nécessaire et suffisante de continuité faible sous une hypothèse de rang constant. Ann. Scuola Norm. Sup. Pisa Cl. Sci. 8 (1981) 68-102.

[10] P. Pedregal, Parametrized measures and variational principles. Birkhäuser, Baston (1997).

[11] P. Suquet, Overall potentials and extremal surfaces of power law or ideally plastic composites. J. Mech. Phys. Solids 41 (1993) $981-1002$.

[12] D.R.S. Talbot and J.R. Willis, Upper and lower bounds for the overall properties of a nonlinear composite dielectric. I. Random microgeometry. Proc. Roy. Soc. London A 447 (1994) 365-384.

[13] D.R.S. Talbot and J.R. Willis, Upper and lower bounds for the overall properties of a nonlinear composite dielectric. II. Periodic microgeometry. Proc. Roy. Soc. London A 447 (1994) 385-396.

[14] L. Tartar, Compensated compactness and applications to partial differential equations. Nonlinerar Analysis and Mechanics: Heriot-Watt Symposium, R. Knops Ed., Longman, Harlow. Pitman Res. Notes Math. Ser. 39 (1979) 136-212.

[15] R. Temam, Navier-Stokes Equations. Elsevier Science Publishers, Amsterdam (1977). 